\title{
General Methods for Suppressing the Light Shift in Atomic Clocks Using Power Modulation
}

\author{
V.I. Yudin $\odot,{ }^{1,2,3,}{ }^{*}$ M. Yu. Basalaev $\odot,{ }^{1,2,3}$ A.V. Taichenachev, ${ }^{1,2}$ J.W. Pollock $\odot,{ }^{4,5}$ Z.L. Newman, ${ }^{4}$ \\ M. Shuker, ${ }^{4}$ A. Hansen, ${ }^{4}$ M.T. Hummon ${ }^{\circledR},{ }^{4}$ R. Boudot, ${ }^{6}$ E.A. Donley ${ }^{\circledR},{ }^{4}$ and J. Kitching ${ }^{4}$ \\ ${ }^{1}$ Novosibirsk State University, ul. Pirogova 1, Novosibirsk 630090, Russia \\ ${ }^{2}$ Institute of Laser Physics SB RAS, pr. Akademika Lavrent'eva 15B, Novosibirsk 630090, Russia \\ ${ }^{3}$ Novosibirsk State Technical University, pr. Karla Marksa 20, Novosibirsk 630073, Russia \\ ${ }^{4}$ National Institute of Standards and Technology, Boulder, Colorado 80305, USA \\ ${ }^{5}$ University of Colorado, Boulder, Colorado 80309-0440, USA \\ ${ }^{6}$ FEMTO-ST, CNRS, UBFC, ENSMM, 26 rue de l'épitaphe, Besancon 25030, France
}

(Received 1 April 2020; revised 21 May 2020; accepted 17 June 2020; published 3 August 2020)

\begin{abstract}
We show that the light shift in atomic clocks can be suppressed using time variation of the interrogation field intensity. By measuring the clock output at two intensity levels, error signals can be generated that simultaneously stabilize a local oscillator to an atomic transition and correct for the shift of this transition caused by the interrogating optical field. These methods are suitable for optical clocks using one- and two-photon transitions, as well as for microwave clocks based on coherent population trapping or direct interrogation. The proposed methods can be widely used both for high-precision scientific instruments and for a wide range of commercial clocks, including chip-scale atomic clocks.
\end{abstract}

DOI: 10.1103/PhysRevApplied.14.024001

\section{INTRODUCTION}

Atomic clocks have had broad impact in both fundamental physics and practical applications. They have enabled, for example, some of the most precise tests of general relativity $[1,2]$ and put stringent limits on the possible variation with time of fundamental constants [3]. In addition, they underlie many aspects of modern technological infrastructure, such as global navigation satellite systems and high-speed telecommunication systems. The current state of the art, problems, and some prospects for the further development of atomic clocks of various types and purposes are well presented in reviews [4-6].

The most important metrological characteristics of an atomic clock are its long-term stability and accuracy. One of the main factors limiting these performance metrics is the ac Stark shift of atomic levels caused by the presence of any optical fields used to probe the atoms. The ac stark shift is particularly problematic for atomic clocks based on coherent population trapping, and optical clocks,

\footnotetext{
*viyudin@mail.ru
}

Published by the American Physical Society under the terms of the Creative Commons Attribution 4.0 International license. Further distribution of this work must maintain attribution to the author(s) and the published article's title, journal citation, and DOI. where optical fields are necessarily present in the interrogation sequence. Understanding, and ultimately suppressing, this light shift is therefore important in improving the long-term frequency stability of these types of references. Particular successes in this direction have been achieved over the past decade for atomic clocks based on Ramsey spectroscopy. This process began in Ref. [7], where the method of so-called hyper-Ramsey spectroscopy was developed, which made it possible to reduce the light shift and its variations for a reference atomic resonance by several orders of magnitude [8,9]. Further development of the hyper-Ramsey approach has used other phase variants to build an error signal [10-16]. This allows for significant improvement in the efficiency of suppression of the light shift in atomic clocks.

Autobalanced Ramsey (ABR) spectroscopy is another effective approach that was experimentally demonstrated in a ${ }^{171} \mathrm{Yb}^{+}$ion clock [17]. This approach was rigorously substantiated and generalized in Ref. [18], and also recently realized in atomic clocks based on coherent population trapping (CPT) [19-21]. In ABR spectroscopy, two Ramsey sequences with different Ramsey times are used. A primary control loop stabilizes the clock frequency as in conventional Ramsey spectroscopy, while a second loop controls an adjustable property of the first and/or second Ramsey pulses, for example, a phase jump of the local oscillator during the Ramsey sequence. An alternative method named combined error signal (CES) spectroscopy 
has recently been proposed [22] and demonstrated [23]. In contrast to the ABR protocol, the Ramsey-CES method uses a single combined error signal, constructed by subtracting the error signals obtained from the two Ramsey subsequences with an appropriate normalization factor (calibration coefficient). This one-loop method offers lightshift mitigation and reduces the complexity of implementing the two-loop control system required for ABR-like protocols at the cost of having to measure or otherwise establish the normalization factor.

Although successful as laboratory demonstrations, these approaches have had no significant effect on the development and improvement of commercial atomic clocks, which typically use continuous wave (cw) spectroscopy rather than Ramsey spectroscopy. The main reason is that, from the viewpoint of commercial instrumentation, reliability and relative simplicity of devices play a critical role and $\mathrm{cw}$ spectroscopy in atomic vapor cells is preferred. Moreover, in a number of important cases Ramsey spectroscopy is impractical because of the short lifetime of the excited state. For example, this is true for modern optical clocks using vapor cells with alkali atoms, such as for onephoton sub-Doppler spectroscopy with counterpropagating waves at the $S \leftrightarrow P$ transition (see, e.g., Ref. [24]); twophoton spectroscopy on the $S \leftrightarrow D$ transition (see, e.g., Refs. [25-28]); and Doppler-free spectroscopy of molecular iodine (see, e.g., Ref. [29]). In addition, in atomic clocks based on Ramsey spectroscopy in atomic beams and fountains (see, e.g., Ref. [30]), the high-efficient ABR and CES methods developed in Refs. [17,18,22] cannot be used, because in these devices the free evolution time cannot be varied to form two Ramsey sequences with different Ramsey times.

While cw spectroscopy is more widely used in commercial clocks at present, general methods to mitigate the light shift in a manner similar to the ABR and CES protocols has not yet been developed. To date, several approaches to the problem of the light shift in cw spectroscopy are known for various types of clocks. The first efforts in this direction were made for microwave CPT clocks, for which the light shift can be suppressed by a suitable choice of the rf modulation index of the laser field [31,32]. Light shifts have also been suppressed in conventional optically pumped microwave clocks by the appropriate choice of the frequency $\omega_{\mathrm{OP}}$ of the optical pumping field [33]. Recently, a method has been proposed that can suppress the light shift for two-photon spectroscopy on the transition $\left(5 s^{2} S_{1 / 2}\right) \leftrightarrow\left(5 d^{2} D_{5 / 2}\right)$ in ${ }^{87} \mathrm{Rb}$ through the use of two interrogating laser fields at different frequencies [34]. Therefore, the development of effective and universal methods of suppressing the light shift for cw spectroscopy of clock transitions is currently a very relevant research topic.

In this paper, we develop two methods for suppressing the light shift and its fluctuation in atomic clocks based on either cw or Ramsey spectroscopy. Both methods use a power modulation (PM) with sequential alternating operations with two different laser powers $P_{1}$ and $P_{2}$. The first method operates with a single combined error signal (PM-CES), constructed by subtracting the error signals obtained from the two subsequences $\left(P_{1}\right.$ and $\left.P_{2}\right)$ with an appropriate normalization factor (calibration coefficient) dependent on the ratio $P_{1} / P_{2}$. The second method uses a two-loop approach to feed back on and stabilize the clock frequency $\omega$ as well as a second (concomitant) parameter $\xi$, which determines the value of the artificial "antishift" of the clock transition that actively autocompensates the original shift (we refer to this as ACS). The operation of PM-ACS consists of the correlated stabilization of both variable parameters $\omega$ and $\xi$, which leads to the light shift cancelation for the clock frequency $\omega$. Another variant of PM-ACS using low frequency harmonic power modulation is also proposed. While the two-loop PM-ACS is more complicated to implement than the single-loop PM-CES, it also requires fewer constraints, as outlined below. Both the PM-CES and the PM-ACS methods can be applied in optical clocks using one-photon and two-photon spectroscopy, as well as in rf clocks based on CPT resonances and optical pumping clocks.

\section{GENERAL DESCRIPTION OF THE LIGHT SHIFT IN ATOMIC CLOCKS}

Let us consider an atomic clock (either optical or microwave) in which the frequency of the local oscillator $\omega$ is stabilized by a reference atomic transition with an unperturbed frequency $\omega_{0}$. For this purpose, a resonant spectroscopic signal with a characteristic linewidth $\gamma$ [see Fig. 1(a)] is used, on the basis of which the dispersionlike error signal $S(\delta)$ is generated in some known way (e.g., by the use of harmonic frequency modulation, frequency jumps, phase jumps) as a function of the detuning $\delta=\omega-\omega_{0}$ [see Fig. 1(b)]. The standard operation of an atomic clock is to use a feedback loop to stabilize the frequency $\omega$ at the zero of the error signal, i.e.,

$$
S(\delta)=0,
$$

which, in the ideal case, corresponds to $\delta=0$, i.e., $\omega=\omega_{0}$. However, under the influence of a laser field, the resonant atomic transition experiences a light shift $\Delta_{P}$ [see Fig. 1(a)], which also manifests in the error signal [see Fig. 1(b)]. In this case, the frequency stabilization can be mathematically represented as

$$
S\left(\delta-\Delta_{P}\right)=0,
$$

which leads to the result

$$
\delta=\Delta_{P} \quad \Rightarrow \quad \omega=\omega_{0}+\Delta_{P}
$$




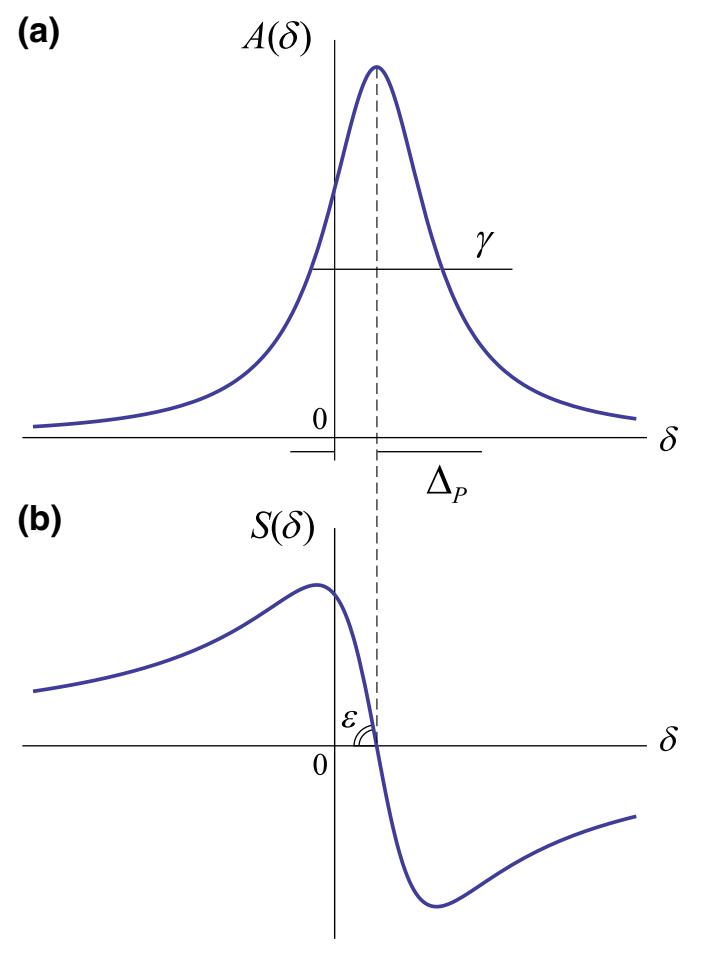

FIG. 1. Illustration of characteristic spectroscopic lineshapes. (a) The resonant lineshape of the spectroscopic signal $A(\delta)$ at the clock transition; (b) the corresponding error signal $S(\delta)$.

describing effects of the light shift on the stabilized frequency. We assume further that the main source of light shift is the ac Stark shift, which negatively affects not only the accuracy of atomic clocks, but also their long-term stability due to temporal variations in the value of $\Delta_{P}(t)$ caused by power and frequency fluctuations of the laser field. Moreover, for rf clocks based on coherent population trapping, additional variations of $\Delta_{P}(t)$ can also occur from fluctuations in the modulation index of the laser field at the operating $\mathrm{rf}$ frequency $\omega$.

Below we consider two methods for suppressing the ac Stark shift and its variations in atomic clocks. We assume that the possible uncontrolled variations of the light shift $\Delta_{P}$ and the laser power $P$ are sufficiently slow, such that these variations are negligible during one measurement cycle used to correct the stabilized frequency $\omega$.

\section{COMBINED ERROR SIGNAL AT POWER MODULATION (PM-CES)}

In this section, we describe a method of the combined error signal (PM-CES). We assume that the atomic clock operates in a time-interleaved manner at two different values of the probe laser field power, $P_{1}$ and $P_{2}$. Moreover, we suppose that the following conditions are fulfilled.
(1) The light shift is linear in the optical power:

$$
\Delta_{P}=c P
$$

Here $c$ is an empirical coefficient.

(2) The slope of the error signal in the line center has a pure power-law dependence on $P$ [see Fig. 1(b)]:

$$
\tan \varepsilon=b P^{\alpha}
$$

Here $b$ and $\alpha$ can be arbitrary. This assumption implies that the system cannot exhibit saturation or power broadening of the spectroscopic transition.

(3) The light shift is much smaller than the linewidth:

$$
\Delta_{P} \ll \gamma .
$$

Let us consider now the error signals $S_{P_{1}}(\delta)$ and $S_{P_{2}}(\delta)$ for two values $P_{1}$ and $P_{2}$. Because of condition (6), near the zero of the error signals, the linear approximation can be used with good accuracy, as shown in Fig. 2(a). Using
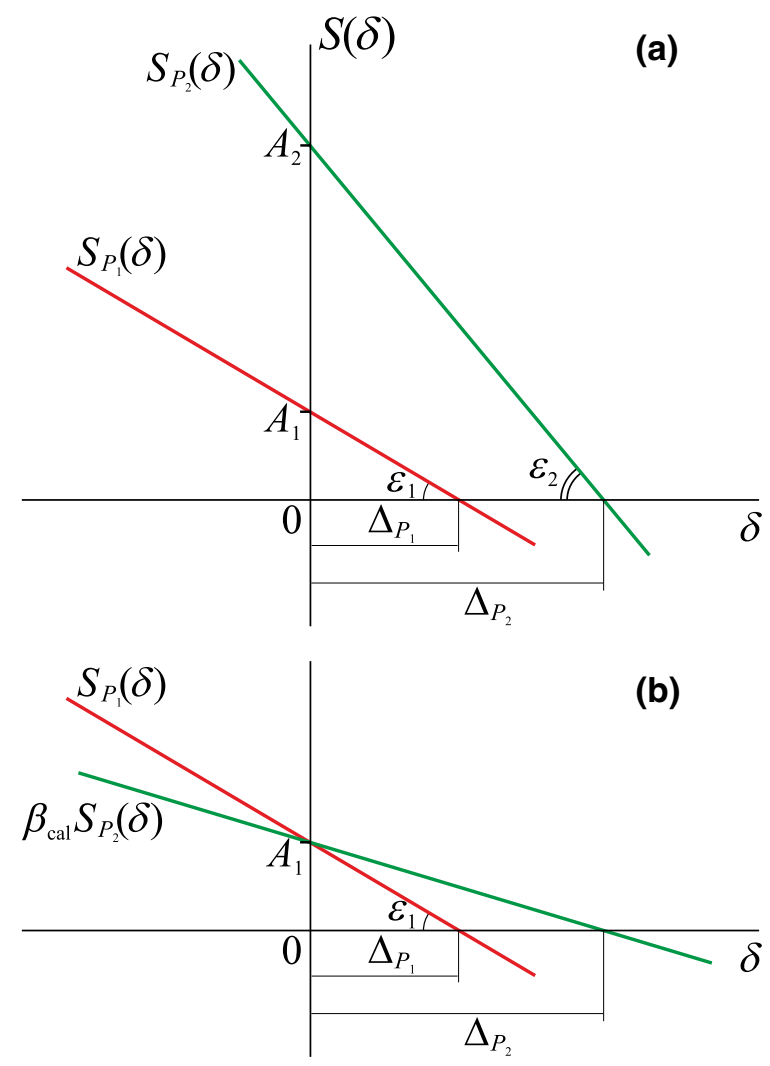

FIG. 2. Illustration of error signals. (a) Linear central sections of the error signals $S_{P_{1}}(\delta)$ (red line) and $S_{P_{2}}(\delta)$ (green line) taking into account light shifts $\Delta_{P_{1}}$ and $\Delta_{P_{2}}$; (b) transformation of upper figure (a) when instead of the dependence $S_{P_{2}}(\delta)$, the product $\beta_{\text {cal }} S_{P_{2}}(\delta)$ is presented (green line), in which the calibration coefficient $\beta_{\text {cal }}$ [see Eq. (8)] is used. 


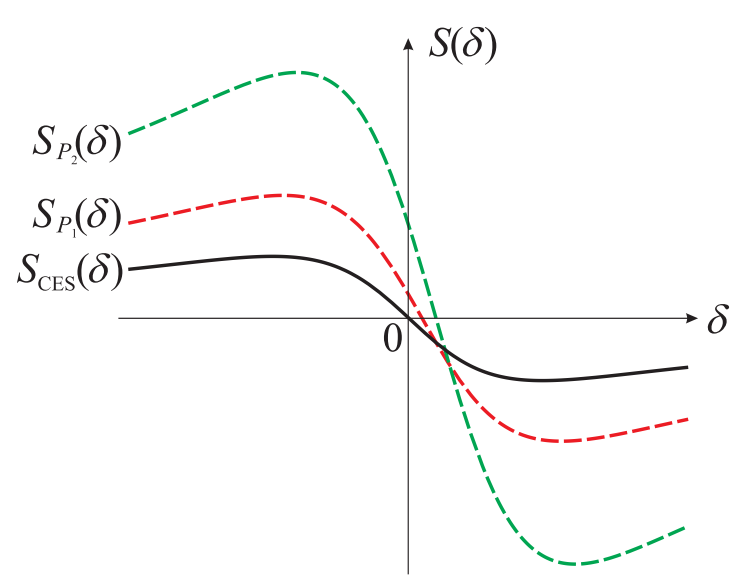

FIG. 3. Illustration showing the absence of a light shift for the combined error signal $S_{\mathrm{CES}}(\delta)$ (black solid line), while the common error signals $S_{P_{1}}(\delta)$ (red dashed line) and $S_{P_{2}}$ (green dashed line) experience light shifts.

well-known trigonometric formulas, we find the ratio of the segments $0 A_{2}$ and $0 A_{1}$ in Fig. 2(a) to be

$$
\frac{0 A_{2}}{0 A_{1}}=\frac{\Delta_{P_{2}} \tan \varepsilon_{2}}{\Delta_{P_{1}} \tan \varepsilon_{1}}=\left(\frac{P_{2}}{P_{1}}\right)^{\alpha+1},
$$

where we used expressions (4) and (5). If we multiply the error signal $S_{P_{2}}(\delta)$ by the calibration coefficient $\beta_{\text {cal }}$, which is inverse to ratio (7),

$$
\beta_{\mathrm{cal}}=\left(\frac{P_{1}}{P_{2}}\right)^{\alpha+1}
$$

then the dependence $\beta_{\mathrm{cal}} S_{P_{2}}(\delta)$ will cross the other error signal $S_{P_{1}}(\delta)$ at the point $A_{1}$ [as shown in Fig. 2(b)], which is located on the vertical axis corresponding to the unshifted frequency, $\delta=0$. As a result, if we construct a combined error signal (PM-CES) as

$$
S_{\mathrm{CES}}(\delta)=S_{P_{1}}(\delta)-\beta_{\mathrm{cal}} S_{P_{2}}(\delta)
$$

then the dependence $S_{\mathrm{CES}}(\delta)$ will cross the horizontal axis at the point $\delta=0$, as shown in Fig. 3. Thus, if we use PMCES (9) to stabilize the frequency $\omega$, as a result, we obtain

$$
S_{\mathrm{CES}}(\delta)=0 \quad \Rightarrow \quad \delta=0 \quad \Rightarrow \quad \omega=\omega_{0}
$$

which means that the atomic clock light shift and its variations are suppressed.

Note that the above result is independent of whether $P_{2}>P_{1}$ or $P_{2}<P_{1}$. Indeed, the clock stability depends on the signal-to-noise ratio, which in our case is determined as

$$
\begin{aligned}
\frac{\left|\tan \varepsilon_{\mathrm{CES}}\right|}{N_{\mathrm{CES}}} & =\frac{\left|\tan \varepsilon_{1}-\beta_{\mathrm{cal}} \tan \varepsilon_{2}\right|}{\sqrt{N_{P_{1}}^{2}+\beta_{\mathrm{cal}}^{2} N_{P_{2}}^{2}}} \\
& =\frac{\left|\tan \varepsilon_{1}-\left(P_{1} / P_{2}\right)^{\alpha+1} \tan \varepsilon_{2}\right|}{\sqrt{N_{P_{1}}^{2}+\left(P_{1} / P_{2}\right)^{2(\alpha+1)} N_{P_{2}}^{2}}},
\end{aligned}
$$

where $\tan \varepsilon_{\mathrm{CES}}$ is the slope of the combined error signal (9); $N_{P_{1}}$ and $N_{P_{2}}$ are noises for $P_{1}$ and $P_{2}$, respectively. It can be easy shown that this ratio does not change with the permutation $P_{1} \rightarrow P_{2}$ and $P_{2} \rightarrow P_{1}$. In addition, by maximizing the signal-to-noise ratio (11), we can determine the optimal power ratio, $P_{2} / P_{1}$. However, such an optimization will depend on the type of spectroscopy (transmission, fluorescence, etc.) as well as on the nature of the dominating noise (e.g., shot noise, classical noise, laser noise, etc.), and therefore it should be considered individually for various experimental conditions.

A schematic diagram for experimental implementation of PM-CES is presented in Fig. 4. The power modulator creates an alternating sequence of two different powers $P_{1}$ and $P_{2}$ of the laser. The frequency of power modulation is much slower than $f_{\text {mod }}$, the modulation frequency used to generate the error signals $S_{P_{1,2}}$ (see Fig. 4, lock-in amplifier). Note that, for example, an acousto-optical modulator or a liquid crystal waveplate followed by a polarizer can be used as the power modulator. Next, before entering the atomic cell, a beam splitter is installed, which splits the laser beam into two. One beam passes through the atomic cell and forms a spectroscopic signal and error signals $S_{P_{1}}(\delta)$ and $S_{P_{2}}(\delta)$ using the first photodetector $\mathrm{PD}_{1}$. In the case of one-photon spectroscopy, this can be a transmission signal, while in the case of two-photon spectroscopy, this can be a fluorescence signal. The second beam is incident on photodetector $\mathrm{PD}_{2}$ and is used to calculate the ratio $P_{1} / P_{2}$, i.e., to determine the calibration coefficient $\beta_{\text {cal }}$ [see Eq. (8)]. The digital block " $N$-box" based on data from the photodetectors $\mathrm{PD}_{1}$ and $\mathrm{PD}_{2}$ generates the combined error signal $S_{\mathrm{CES}}(\delta)$ [see Eq. (9)], which is then used to stabilize the frequency $\omega$ of the local oscillator (LO) by the condition $S_{\mathrm{CES}}(\delta)=0$.

PM-CES is a quite general method suitable for various types of spectroscopy. For example, for one-photon spectroscopy in counterpropagating waves and in the weak saturation regime of the clock transition, the signal size is linear in power and we have $\alpha=1$. In this case, the calibration coefficient is

$$
\beta_{\mathrm{cal}}^{(1-\mathrm{ph})}=\left(\frac{P_{1}}{P_{2}}\right)^{2} .
$$

In the case of a fluorescence signal for two-photon spectroscopy, where the signal size scales with the power 


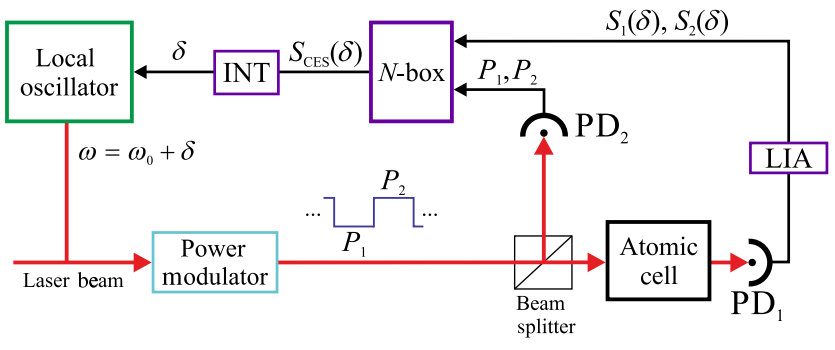

FIG. 4. The general scheme of the experimental implementation of the PM-CES method. INT denotes the integrator; LIA denotes the lock-in amplifier; PD denotes the photodetector.

squared, $\alpha=2$, the calibration coefficient is

$$
\beta_{\mathrm{cal}}^{(2-\mathrm{ph})}=\left(\frac{P_{1}}{P_{2}}\right)^{3}
$$

PM-CES can also be used in CPT clocks as well as for optical pumping clocks. In general, the success of the PMCES method is directly related to how accurately the basic conditions in Eqs. (4)-(6) are fulfilled in experiments.

In addition, in some cases, to obtain information about the laser power $P$, we can also use the first photodetector $\mathrm{PD}_{1}$. In this case, the presence of a second photodetector $\mathrm{PD}_{2}$ is optional, which provides some technical simplification. Note also that the PM-CES method presented above can be considered as a PM analog of the Ramsey-CES method developed in Ref. [22].

\section{AUTOCOMPENSATION OF THE LIGHT SHIFT (PM-ACS)}

In this section, we develop a method to autocompensate the light shift (ACS) in spectroscopy using a power modulation (PM-ACS). Unlike the PM-CES method developed in the previous section, $\mathrm{PM}-\mathrm{ACS}$ only requires the linearity of the light shift, Eq. (4), while the other two conditions, Eqs. (5) and (6), do not matter. In PM-ACS, we assume the use of an additional frequency shifter that, for any laser power $P$, allows us to shift the frequency of the local oscillator $\omega$ by the value $\xi P$, where $\xi$ is a well-controlled and variable parameter. This shift

$$
\Delta_{\mathrm{ACS}}=\xi P
$$

plays the role of an artificial antishift, which allows us to completely compensate for the actual light shift $\Delta_{P}=c P$ and its fluctuations. Indeed, taking into account the actual shift $\Delta_{P}$ and the artificial antishift $\Delta_{\mathrm{ACS}}$, the result of the frequency stabilization with the use of the error signal can be represented as a solution of the equation

$$
S_{P}(\delta, \xi)=S_{P}\left(\delta+\Delta_{\mathrm{ACS}}-\Delta_{P}\right)=S_{P}(\delta+\xi P-c P)=0
$$

for an unknown $\delta$. Then, for $\xi=c$, we obtain $\delta=0$, i.e., $\omega=\omega_{0}$.

Below we present the implementation of the PM-ACS method in two different modifications: for stepwise and harmonic modulations of the laser power.

\section{A. PM-ACS for stepwise modulation of power}

We assume that the atomic clock operates at two different values of the probe laser field power $P_{1}$ and $P_{2}$. The PM-ACS method consists of the following repeating cycles. For interrogation with the laser power $P_{1}$, the parameter $\xi$ is fixed, and we stabilize the variable detuning $\delta$ (i.e., LO frequency $\omega$ ) at the zero point of the error signal: $S_{P_{1}}\left(\delta, \xi_{\text {fixed }}\right)=0$. The operation of this servo loop can be presented as the recurrent sequence

$$
\delta_{n}=\delta_{n-1}+r S_{P_{1}}\left(\delta_{n-1}, \xi_{\text {fixed }}\right),
$$

where $r$ is a feedback factor for the frequency loop and $n$ is the step index counter for the sequence. After this procedure, we switch to interrogation with power $P_{2}$, where we fix the previously obtained detuning $\delta$ and stabilize the variable parameter $\xi$ at the zero point of the second error signal: $S_{P_{2}}\left(\delta_{\text {fixed }}, \xi\right)=0$. The operation of this second servo loop can be presented as another recurrent sequence

$$
\xi_{m}=\xi_{m-1}+q S_{P_{2}}\left(\delta_{\text {fixed }}, \xi_{m-1}\right),
$$

where $q$ is a feedback factor for the $\xi$-loop and $m$ is the step index counter for the second servo loop.

If we continue these cycles then the final result consists of the stabilization of both parameters, $\delta=\bar{\delta}_{\text {clock }}$ and $\xi=$ $\bar{\xi}$, which corresponds to the solution of the two-equation system

$$
\begin{aligned}
& S_{P_{1}}(\delta, \xi)=S_{P_{1}}\left(\delta+\xi P_{1}-c P_{1}\right)=0 \\
& S_{P_{2}}(\delta, \xi)=S_{P_{2}}\left(\delta+\xi P_{2}-c P_{2}\right)=0
\end{aligned}
$$

for two unknowns $\delta$ and $\xi$. The value $\bar{\delta}_{\text {clock }}$ describes the frequency shift of the atomic clock. Assuming that $S_{P}(x) \propto$ $x$ for $x \approx 0$, system (18) is equivalent to the system

$$
\begin{aligned}
& \delta+(\xi-c) P_{1}=0 \\
& \delta+(\xi-c) P_{2}=0
\end{aligned}
$$

which has the solution

$$
\delta=0, \quad \xi=c .
$$

Thus, we have shown that the PM-ACS method always leads to zero shift of the stabilized frequency $\omega$ in an atomic clock, $\bar{\delta}_{\text {clock }}=0$.

Note that in the general case the convergence of the joint iterative procedures (16) and (17) to the values (20) 


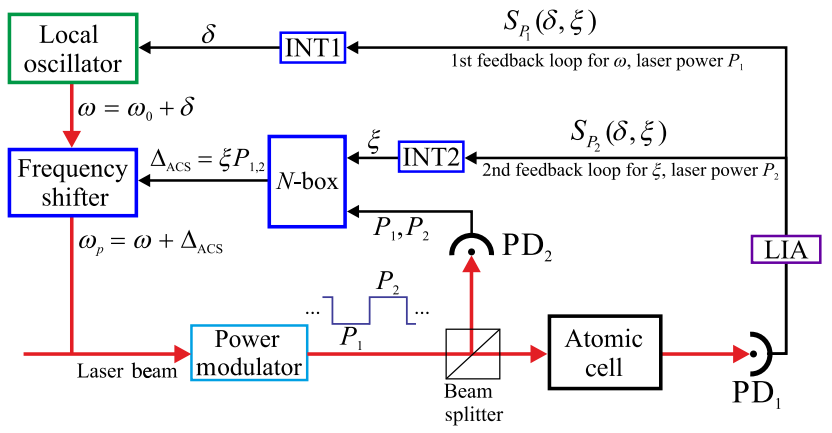

FIG. 5. The general scheme of the experimental implementation of the cw-ACS method for stepwise modulation of power.

depends on the initial values $\delta_{0}$ and $\xi_{0}$ as well as on the feedback coefficients $r$ and $q$. For the initial conditions $\left|\delta_{0}\right| \ll \min \left\{\gamma_{P_{1}}, \gamma_{P_{2}}\right\}$ and $\left|\xi_{0}-c\right| P_{1,2} \ll \gamma_{P_{1,2}}$, the fastest convergence of iterations (16) and (17) is carried out for the feedback coefficients

$$
\begin{aligned}
r & =-\left[\left.\partial_{\delta} S_{P_{1}}(\delta, \xi)\right|_{\delta=0, \xi=c}\right]^{-1}, \\
q & =-\left[\left.\partial_{\xi} S_{P_{2}}(\delta, \xi)\right|_{\delta=0, \xi=c}\right]^{-1},
\end{aligned}
$$

where $\partial_{\delta}$ and $\partial_{\xi}$ denote the differential operators $\partial_{\delta}=\partial / \partial \delta$ and $\partial_{\xi}=\partial / \partial \xi$, respectively; $\gamma_{P_{j}}$ is the linewidth for the power $P_{j}(j=1.2)$. In practice, the optimal values $\delta_{0}, \xi_{0}$, $r$, and $q$ are determined experimentally.

In Fig. 5 we show a schematic diagram of the implementation of the PM-ACS method, which has two feedback loops: a main loop to stabilize the local oscillator detuning $\delta$, and an auxiliary loop to stabilize the scale factor $\xi$ for the artificial antishift $\Delta_{\text {ACS }}$. The circuit element labeled " $N$ box" in Fig. 5 is a multiplier that implements the product $\xi P$. In the absence of the auxiliary loop $(\xi=0)$, the main loop locks the local oscillator to the light-shifted atomic resonance, $\omega_{0}+c P_{1,2}$. When the auxiliary loop is enabled, feedback from the error signal $S_{P_{2}}(\delta, \xi)$ drives the scale factor $\xi$ to be nonzero, and correspondingly causes the probe frequency $\omega_{p}=\omega+\xi P_{2}$ to be locked to the lightshifted resonance, $\omega_{0}+c P_{2}$. This condition, when applied to both operating powers, $P_{1}$ and $P_{2}$, is equivalent to Eqs. (19)-(20).

Note that the continuous use of two feedback loops (for $P_{1}$ and $P_{2}$ ) will lead to some decrease of the short-term stability, because it increases the length of each cycle of frequency stabilization. This can be remedied in the following way. In the initial period of frequency stabilization, we use the two-loop PM-ACS method. This step allows us to determine the value of the concomitant parameter $\bar{\xi}$ with satisfactory accuracy. Then the procedure of long-term frequency stabilization can be done by only one feedback loop for the error signal $S_{P_{1}}(\delta, \bar{\xi})$ using the previously determined parameter $\bar{\xi}$. Moreover, we can regularly (but rarely) use the two-loop PM-ACS again to remeasure $\bar{\xi}$ and correct any long-term drift. On the one hand, this approach allows for the regular adjustment of the parameter $\bar{\xi}$ (to eliminate, for example, an influence of possible slow variations of the characteristics of the second photodetector $\mathrm{PD}_{2}$ ). On the other hand, because the measurement is intermittent, it will not lead to significant reduction of the signal-to-noise ratio and corresponding degradation of the long-term frequency stability. The choice of the ratio of $P_{1}$ to $P_{2}$ is an additional control that can be used to optimize the process.

PM-ACS is a general method useful for a variety of clock laser spectroscopies, including optical clocks using one-photon and two-photon spectroscopy and microwave clocks based on optical pumping or CPT. Note that the amount the light shift can be suppressed using the PMACS method is directly related to how accurately the linearity condition in Eq. (4) is met, although this method can easily be adapted to the case of an arbitrary nonlinear dependence of the actual shift $\Delta_{P}$ on the power $P$. Therefore, a detailed study of light shifts for various atomic clocks would be useful in order to determine the operating modes in which the linearity $\Delta_{P} \propto P$ is best fulfilled, because this regime is most suitable from a practical viewpoint (e.g., in the case of commercial production of atomic clocks using the PM-ACS method).

We also note that the PM-ACS method can be considered conceptually as a PM analog of one of the variants of generalized autobalanced Ramsey spectroscopy given in Refs. [18,21], in which an additional frequency shift was used as the concomitant parameter.

\section{B. PM-ACS for low frequency harmonic modulation of power}

In Fig. 6 we show a schematic diagram of the implementation of another version of PM-ACS using low-frequency harmonic modulation of the laser power:

$$
P(t)=P_{0}[1+M \sin (v t)]
$$

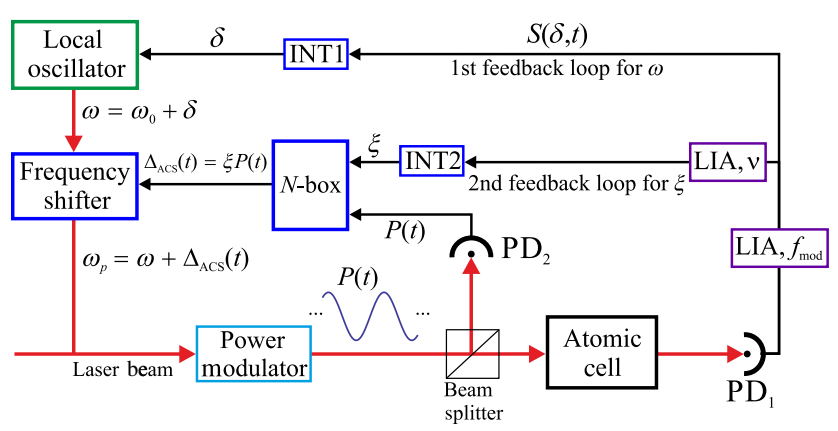

FIG. 6. The general scheme of the experimental implementation of the PM-ACS method for low-frequency harmonic modulation of laser power (22). 
Here $M<1$. In this case, both the actual shift $\Delta_{P}(t)=$ $c P(t)$ and the artificial antishift $\Delta_{\mathrm{ACS}}(t)=\xi P(t)$ become time dependent according to a harmonic law with a frequency $v$. Note that the power modulation frequency $v$ is much lower than the frequency $f_{\text {mod }}$ of the frequency deviation used to generate the error signal $S(\delta)$, as in Ref. [32] for a CPT clock. Additionally, we require that the bandwidth of the feedback to the local oscillator be much lower than the power modulation frequency $v$.

The operation of the scheme in Fig. 6 is the following. The effect of power modulation in Eq. (22) is monitored as modulation on the error signal $S(\delta, t)$ at the frequency $v$. This low-frequency modulation is observed on the error signal because a change in the total optical power produces a corresponding change in the atomic resonance frequency due to both the actual light shift $\Delta_{P}(t)$ and the artificial antishift $\Delta_{\mathrm{ACS}}(t)$ :

$$
\Delta_{P}(t)-\Delta_{\mathrm{ACS}}(t)=(c-\xi) P(t) .
$$

Then, the condition $\xi=c$ at which the power shift contribution in Eq. (23) becomes zero occurs when the $v$ frequency modulation on the error signal vanishes.

\section{CONCLUSION}

We describe two different methods for suppressing the light shift and its variation in atomic clocks based on either cw or Ramsey spectroscopy. The first method (PM-CES) uses only one feedback loop to stabilize the LO frequency but requires the measurement of a calibration factor to create a suitable error signal. The second method (PMACS) requires two loops but has fewer restrictions on the size of the light sift and nature of the error signal. These methods are general in that they can be applied to optical clocks using one-photon and two-photon spectroscopy, as well as microwave clocks based on CPT resonances and optical pumping. The implementation of the PM-CES and PM-ACS techniques can in principle lead to significant improvement of the accuracy and long-term stability for high-precision and commercial atomic clocks.

In addition, although the schemes depicted in Figs. 4 and 5 are more related to the $\mathrm{cw}$ spectroscopy, we believe that the corresponding schemes for Ramsey spectroscopy (in an atomic beam or fountain) will not cause difficulties.

\section{ACKNOWLEDGMENTS}

We thank Sihong Gu, Leo Hollberg, and Joseph Christesen for useful discussions and Yun-Jhih Chen and Abijith Kowligy for helpful comments on the manuscript. This work was supported by the Russian Scientific Foundation (Grant No. 16-12-10147). V. I. Yudin and A. V. Taichenachev were also supported by the Russian Foundation for Basic Research (Grants No. 20-02-00505, No.
18-02-00822, and No. 20-52-12024) and Ministry of Education and Science of the Russian Federation (Grant No. FSUS-2020-0036). R. Boudot was supported by the NIST Guest Researcher program, Délégation Générale de l'Armement (DGA) and Agence Nationale de la Recherche (ANR) in the frame of the LabeX FIRST-TF (Grant No. ANR 10-LABX-0048), EquipX Oscillator-IMP (Grant No. ANR 11-EQPX-0033) and ASTRID PULSACION (Grant No. ANR-19-ASTR-0013-01) projects. This work is also partially funded by NIST.

[1] P. Delva, N. Puchades, E. Schönemann, F. Dilssner, C. Courde, S. Bertone, F. Gonzalez, A. Hees, Ch. Le PoncinLafitte, F. Meynadier, R. Prieto-Cerdeira, B. Sohet, J. Ventura-Traveset, and P. Wolf, Gravitational Redshift Test Using Eccentric Galileo Satellites, Phys. Rev. Lett. 121, 231101 (2018).

[2] S. Herrmann, F. Finke, M. Lulf, O. Kichakova, D. Puetzfeld, D. Knickmann, M. List, B. Rievers, G. Giorgi, C. Gunther, H. Dittus, R. Prieto-Cerdeira, F. Dilssner, F. Gonzalez, E. Schönemann, J. Ventura-Traveset, and C. Lammerzahl, Test of the Gravitational Redshift with Galileo Satellites in an Eccentric Orbit, Phys. Rev. Lett. 121, 231102 (2018).

[3] M. S. Safronova, The search for variation of fundamental constants with clocks, Ann. Phys. 531, 1800364 (2019).

[4] V. Shah and J. Kitching, Advances in coherent population trapping for atomic clocks, Adv. At. Mol. Opt. Phys. 59, 21 (2010).

[5] N. Poli, C. W. Oates, P. Gill, and G. M. Tino, Optical atomic clocks, Rivista Del Nuovo Cimento 36, 555 (2013).

[6] A. D. Ludlow, M. M. Boyd, J. Ye, E. Peik, and P. O. Schmidt, Optical atomic clocks, Rev. Mod. Phys. 87, 637 (2015).

[7] V. I. Yudin, A. V. Taichenachev, C. W. Oates, Z. W. Barber, N. D. Lemke, A. D. Ludlow, U. Sterr, Ch. Lisdat, and F. Riehle, Hyper-Ramsey spectroscopy of optical clock transitions, Phys. Rev. A 82, 011804(R) (2010).

[8] N. Huntemann, B. Lipphardt, M. Okhapkin, Chr. Tamm, E. Peik, A. V. Taichenachev, and V. I. Yudin, Generalized Ramsey Excitation Scheme with Suppressed Light Shift, Phys. Rev. Lett. 109, 213002 (2012).

[9] N. Huntemann, C. Sanner, B. Lipphardt, Chr. Tamm, and E. Peik, Single-Ion Atomic Clock with $3 \times 10^{-18}$ Systematic Uncertainty, Phys. Rev. Lett. 116, 063001 (2016).

[10] R. Hobson, W. Bowden, S. A. King, P. E. G. Baird, I. R. Hill, and P. Gill, Modified hyper-Ramsey methods for the elimination of probe shifts in optical clocks, Phys. Rev. A 93, 010501(R) (2016).

[11] T. Zanon-Willette, S. Almonacil, E. de Clercq, A. D. Ludlow, and E. Arimondo, Quantum engineering of atomic phase shifts in optical clocks, Phys. Rev. A 90, 053427 (2014).

[12] T. Zanon-Willette, E. de Clercq, and E. Arimondo, Probe light-shift elimination in generalized hyper-Ramsey quantum clocks, Phys. Rev. A 93, 042506 (2016).

[13] V. I. Yudin, A. V. Taichenachev, M. Yu. Basalaev, and T. Zanon-Willette, Synthetic frequency protocol for Ramsey 
spectroscopy of clock transitions, Phys. Rev. A 94, 052505 (2016).

[14] T. Zanon-Willette, V. I. Yudin, and A. V. Taichenachev, Generalized hyper-Ramsey resonance with separated oscillating fields, Phys. Rev. A. 92, 023416 (2015).

[15] T. Zanon-Willette, R. Lefevre, A. V. Taichenachev, and V. I. Yudin, Universal interrogation protocol with zero probe-field-induced frequency shift for quantum clocks and high-accuracy spectroscopy, Phys. Rev. A 96, 023408 (2017).

[16] T. Zanon-Willette, R. Lefevre, R. Metzdorff, N. Sillitoe, S. Almonacil, M. Minissale, E. de Clercq, A. V. Taichenachev, V. I. Yudin, and E. Arimondo, Composite laser-pulses spectroscopy for high-accuracy optical clocks: A review of recent progress and perspectives, Rep. Prog. Phys. 81, 094401 (2018).

[17] Ch. Sanner, N. Huntemann, R. Lange, Ch. Tamm, and E. Peik, Autobalanced Ramsey Spectroscopy, Phys. Rev. Lett. 120, 053602 (2018).

[18] V. I. Yudin, A. V. Taichenachev, M. Yu. Basalaev, T. Zanon-Willette, J. W. Pollock, M. Shuker, E. A. Donley, and J. Kitching, Generalized Autobalanced Ramsey Spectroscopy of Clock Transitions, Phys. Rev. Appl. 9, 054034 (2018).

[19] M. Abdel Hafiz, G. Coget, M. Petersen, C. Rocher, S. Guérandel, T. Zanon-Willette, E. de Clercq, and R. Boudot, Toward a High-Stability Coherent Population Trapping Cs Vapor-Cell Atomic Clock Using Autobalanced Ramsey Spectroscopy, Phys. Rev. Appl. 9, 064002 (2018).

[20] M. Abdel Hafiz, G. Coget, M. Petersen, C. Calosso, S. Guérandel, E. de Clercq, and R. Boudot, Symmetric autobalanced Ramsey interrogation for high-performance coherent-population-trapping vapor-cell atomic clock, Appl. Phys. Lett. 112, 244102 (2018).

[21] M. Shuker, J. W. Pollock, R. Boudot, V. I. Yudin, A. V. Taichenachev, J. Kitching, and E. A. Donley, Ramsey Spectroscopy with Displaced Frequency Jumps, Phys. Rev. Lett. 122, 113601 (2019).

[22] V. I. Yudin, A. V. Taichenachev, M. Yu. Basalaev, T. Zanon-Willette, T. E. Mehlstäubler, R. Boudot, J. W. Pollock, M. Shuker, E. A. Donley, and J. Kitching, Combined error signal in Ramsey spectroscopy of clock transitions, New J. Phys. 20, 123016 (2018).
[23] M. Shuker, J. W. Pollock, R. Boudot, V. I. Yudin, A. V. Taichenachev, J. Kitching, and E. A. Donley, Reduction of light shifts in Ramsey spectroscopy with a combined error signal, Appl. Phys. Lett. 114, 141106 (2019).

[24] M. Abdel Hafiz, G. Coget, E. De Clercq, and R. Boudot, Doppler-free spectroscopy on the $\mathrm{Cs}_{1}$ line with a dualfrequency laser, Opt. Lett. 41, 2982 (2016).

[25] C. Perrella, P. S. Light, J. D. Anstie, T. M. Stace, F. Benabid, and A. N. Luiten, High-resolution two-photon spectroscopy of rubidium within a confined geometry, Phys. Rev. A 87, 013818 (2013).

[26] C. Perrella, P. S. Light, J. D. Anstie, F. N. Baynes, F. Benabid, and A. N. Luiten, Two-color rubidium fiber frequency standard, Opt. Lett. 38, 2122 (2013).

[27] K. W. Martin, G. Phelps, N. D. Lemke, M. S. Bigelow, B. Stuhl, M. Wojcik, M. Holt, I. Coddington, M. W. Bishop, and J. H. Burke, Compact Optical Atomic Clock Based on a Two-Photon Transition in Rubidium, Phys. Rev. Appl. 9, 014019 (2018).

[28] K. W. Martin, B. Stuhl, J. Eugenio, M. S. Safronova, G. Phelps, J. H. Burke, and N. D. Lemke, Frequency shifts due to Stark effects on a rubidium two-photon transition, Phys. Rev. A 100, 023417 (2019).

[29] T. Schuldt, K. Döringshoff, E. V. Kovalchuk, A. Keetman, J. Pahl, A. Peters, and C. Braxmaier, Development of a compact optical absolute frequency reference for space with 10-15 instability, Appl. Opt. 56, 1101 (2017).

[30] F. Riehle, Frequency Standards: Basics and Applications (Wiley-VCH, Weinheim, 2005).

[31] M. Zhu and L. S. Cutler, in Proceedings of 32th Annual Precise Time and Time Interval Systems and Applications Meeting (Institute of Navigation, Inc., Reston, Virginia, 2000), p. 311.

[32] V. Shah, V. Gerginov, P. D. D. Schwindt, S. Knappe, L. Hollberg, and J. Kitching, Continuous light-shift correction in modulated coherent populationtrapping clocks, Appl. Phys. Lett. 89, 151124 (2006).

[33] B. H. McGuyer, Y.-Y. Jau, and W. Happer, Simple method of light-shift suppression in optical pumping systems, Appl. Phys. Lett. 94, 251110 (2009).

[34] V. Gerginov and K. Beloy, Two-Photon Optical Frequency Reference with Active ac Stark Shift Cancellation, Phys. Rev. Appl. 10, 014031 (2018). 